\title{
The classical-quantum correspondence of a kicked particle in an infinite potential well
}

\author{
D. Kilbane ${ }^{\text {a,b,c }}$, A. Cummings ${ }^{\text {a }}$, G. O’Sullivan ${ }^{\text {a }}$, D.M. Heffernan ${ }^{\text {b,c,** }}$ \\ ${ }^{a}$ School of Physics, University College Dublin, Ireland \\ ${ }^{\mathrm{b}}$ Department of Mathematical Physics, National University of Ireland, Maynooth, Ireland \\ ${ }^{c}$ School of Theoretical Physics, Dublin Institute for Advanced studies, Dublin 4, Ireland
}

Accepted 15 December 2005

\begin{abstract}
The classical-quantum correspondence of a periodically kicked particle in a 1-D infinite potential well is investigated. Stroboscopic state space portraits are presented for various kick strengths and a classical diffusion study reveals anomalous behaviour and the presence of both regular islands of stability and accelerator modes. Quantum diffusion is subsequently studied and the quantum diffusion coefficient is found to mimic the classical diffusion coefficient by rescaling $k$. Wigner and Husimi distribution functions are derived and comparisons are made between the classical stroboscopic state space portraits and these quantum quasi-probability distribution functions.
\end{abstract}

(c) 2006 Elsevier Ltd. All rights reserved.

\section{Introduction}

To date, most studies of quantum chaos have been based on quantum systems whose classical counterparts obey the Kolmogorov-Arnol'd-Moser (KAM) theorem. Much of the work has concentrated on smooth Hamiltonian systems. If an integrable system is perturbed (by increasing an external or driven parameter) the invariant curves gradually break up and diffusion can take place leading to global chaos. Examples of such systems are the kicked rotor [1,2] and quantum billiards [3]. As well as these systems, there exists another class of physically important systems, which is nonKAM. In particular, if the perturbation is not sufficiently smooth or even discontinuous the KAM theorem breaks down. Large-scale chaos may instantaneously develop in the system. Examples of such systems are the kicked harmonic oscillator ([4-12] and references therein), and discontinuous perturbed twist maps by Borgonovi [13].

Another example of a non-KAM system is the model of a kicked particle in an infinite potential well introduced by $\mathrm{Hu}$ et al. [14]. For a small perturbation $(K)$ the classical phase space displays a stochastic web structure, and the diffusion coefficient scales as $D \propto K^{2.5}$, while for large perturbations, $D \propto K^{2}$. In [15,16], a discontinuous generalization of the standard map, which arises naturally as the dynamics of a periodically kicked particle in an infinite potential well, was examined. By introducing competing length scales, namely the width of the well and the wavelength of the external field, large-scale diffusion occurs even for small external field strengths. In [17] accelerator modes of a particle confined

\footnotetext{
* Corresponding author. Tel.: +353 1 7083775; fax: +3531 7083967.

E-mail address: dmh@thphys.may.ie (D.M. Heffernan).
} 
in an infinite potential well and subjected to a periodic pulsed field were studied. It was found that accelerator mode assisted transport is greatly enhanced when low order resonances are exposed at the border of chaos. In the same work transport in the corresponding quantum system is studied using a time evolved wavepacket [17].

In this work the classical-quantum correspondence of a kicked particle in an infinite potential well is investigated using the Wigner and Husimi distribution functions. Stroboscopic state space portraits are presented for varying kick strengths in Section 2 along with a classical diffusion study which led to the observation of accelerator modes and anomalous diffusion. In Section 3 the quantum dynamics of the system are investigated with particular emphasis on a quantum energy and diffusion study. Derivations of the quasi-probability distribution functions for the system, namely the Wigner and Husimi distribution functions are presented in Section 4. Concluding remarks are made in Section 5.

\section{Classical dynamics of a kicked particle in a 1-D infinite potential well}

The Hamiltonian of this system introduced by $\mathrm{Hu}$ et al. [14] is defined as

$$
H=\frac{p^{2}}{2 m}+V_{0}(q)+k \cos (q+\alpha) \sum_{n=-\infty}^{\infty} \delta(t-n T),
$$

where

$$
V_{0}(q)= \begin{cases}0, & 0<q<\pi \\ \infty, & \text { otherwise }\end{cases}
$$

$\alpha=1$ is a phase shift introduced to break the parity symmetry of the well [14], $T=1$ is the period of the kick and $k$ is the kick strength. The classical area-preserving mapping for this system is given by

$$
p_{n+1}=p_{n}+k \sin \left(q_{n}+\alpha\right), \quad q_{n+1}=q_{n}+p_{n+1},
$$

where boundary conditions restrict the particles motion to between 0 and $\pi$. The system has the following window of stability $0<-k \cos \left(q_{n}+\alpha\right)<4$ [18]. The fixed point $(q, p)=(0.57,0)$ is hyperbolic for $k>0[18,15]$. For $0<k<4$ the fixed point at $(q, p)=(2.14,0)$ is elliptic.

\subsection{Stroboscopic state space portraits}

Fig. 1 shows the stroboscopic state space for a range of values of the kick strength $(k>0)$ [initial conditions are $\left(q_{0}=0.012,-0.3<p_{0}<0.3\right)$ and $\left.\left(0.012<q_{0}<\pi, p_{0}=0.1\right)\right]$ and iterated for 10,000 steps. The main characteristic of this system is the existence of stochastic webs in the classical phase space for any $k>0$ [14].

Various regions of the classical phase space are shown in Figs. 2-4. For a small perturbation, $k=0.01$, many cantori are present which act as barriers to diffusion. As $k$ increases to $k=0.1$ the stochastic layer widens and the gaps between cantori grow so that the cantori are no longer substantial barriers to diffusion but partial barriers. For $k=5$ almost all of the cantori have disintegrated and global diffusion can occur, however some islands of stability still remain present.

\subsection{Classical diffusion and accelerator modes}

There exist two different regions of classical diffusion as observed by Hu et al. [14]. For $k \gg 1, D \propto k^{2}$ whereas for $k \ll 1, D \propto k^{2.5}$ [14]. In calculating the diffusion coefficient for a given $k$, we have taken a grid of $N \times N(N=50)$, initial conditions $\left(0<q_{0}<\pi,-0.3<p_{0}<0.3\right)$. All initial trajectories are evolved 10,000 times and average energy values are taken over the 2500 starting points for each time step $n$. The diffusion coefficient is defined as $D \equiv\left\langle E_{n}\right\rangle / n$ (a linear fit to $\left\langle E_{n}\right\rangle$ versus $n$ gives a slope $D$ ). At many points in the region $k=1 \rightarrow 25$ it was not possible to perform a linear fit to $\left\langle E_{n}\right\rangle$ versus $n$. An example of trajectories in this region (after 200 iterations) are shown for $k=2.5$ in Fig. 5. The structures that cause these deviations are accelerator modes [2]. The main family of accelerator modes occur in the region $(2 \pi \eta)<k<\sqrt{(2 \pi \eta)^{2}+16}$ (for integer $\eta$ ), where the corresponding accelerator mode hops monotonically by $2 \pi \eta$ in momentum per iteration [2]. Regular stability islands tend to trap trajectories at a fixed momentum, leading to reduced momentum transport, whereas accelerator modes lead to enhanced momentum transport [19]. This subdiffusive or superdiffusive behaviour due to island structures is referred to as anomalous diffusion $[2,20]$. By this we mean that $p^{2}$ grows with time as $n^{\mu}$ with fractional $\mu$, where $\mu=1$ is diffusive, while $\mu=2$ is considered ballistic [21]. In this regime the kinetic energy is $E_{n} \sim n^{\mu}$, where the transport exponent $\mu \neq 1$ for anomalous diffusion as shown in Fig. 5. 

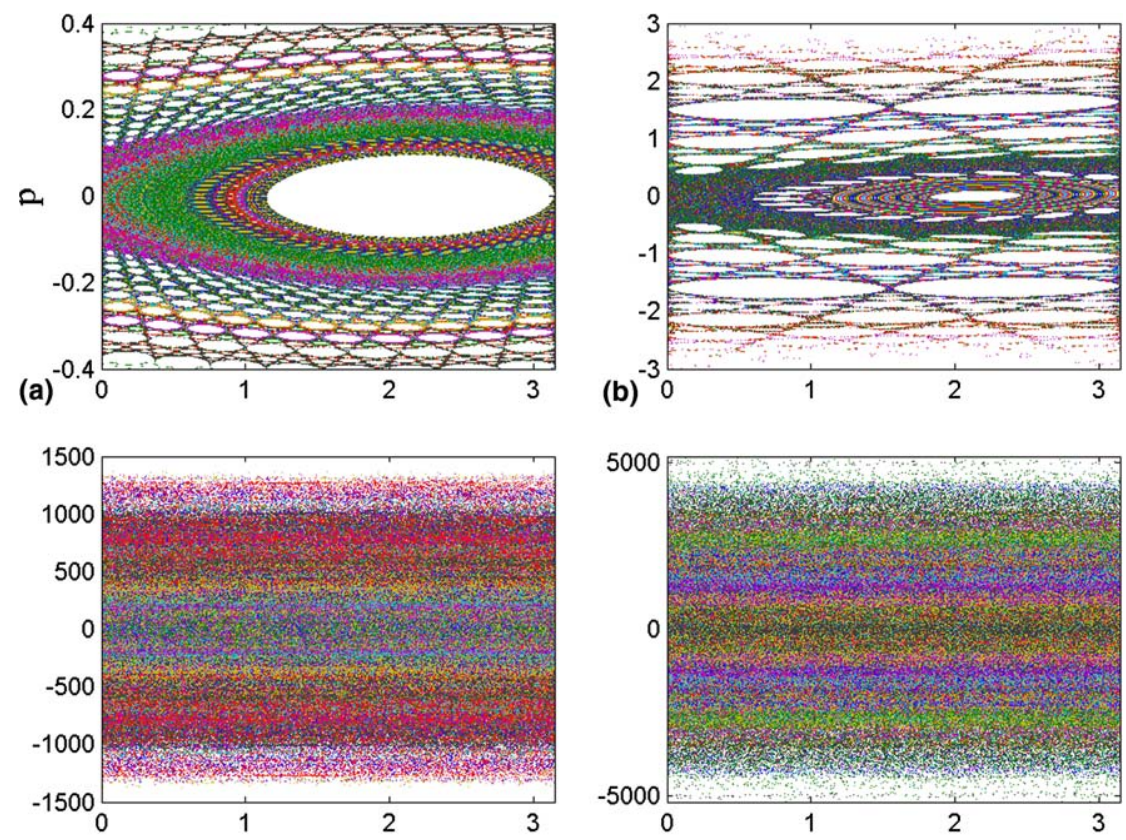

(c)



Fig. 1. The classical stroboscopic state space at (a) $k=0.01$, (b) $k=0.1$, (c) $k=5$ and (d) $k=25$. The stochastic web structure is clearly seen even for very small $k=0.01$.
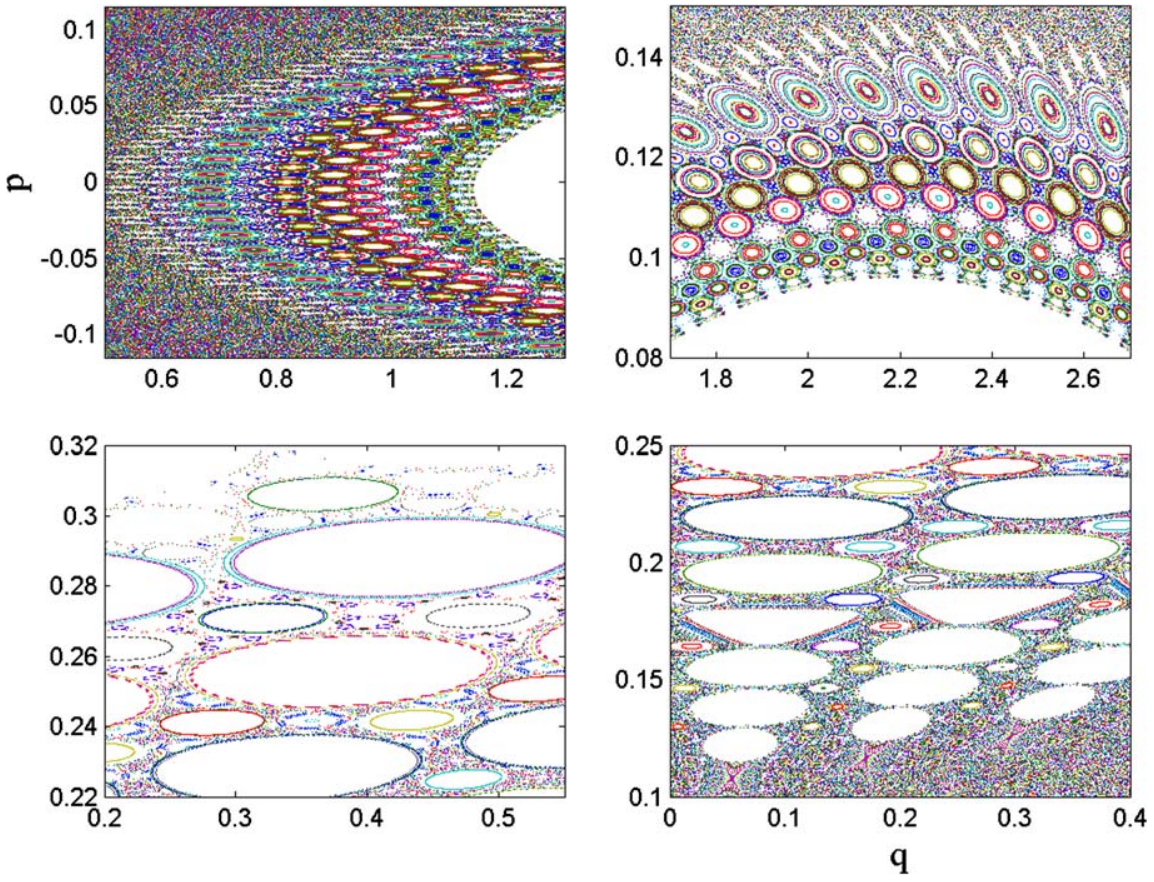

Fig. 2. The classical stroboscopic state space at $k=0.01$, each subplot is a zoom of different regions in the main plot in Fig. 1 . 

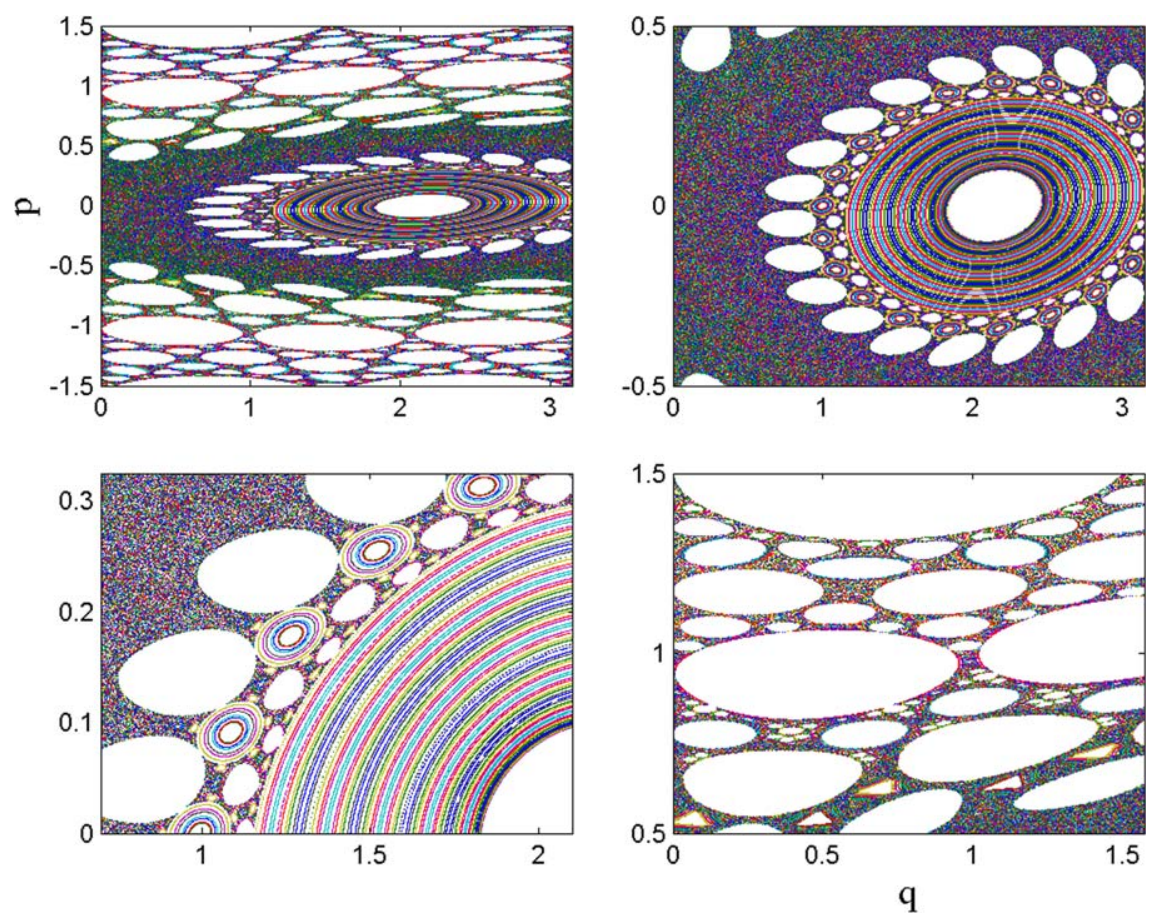

Fig. 3. The classical stroboscopic state space at $k=0.1$, each subplot is a zoom of different regions in the main plot in Fig. 1 .
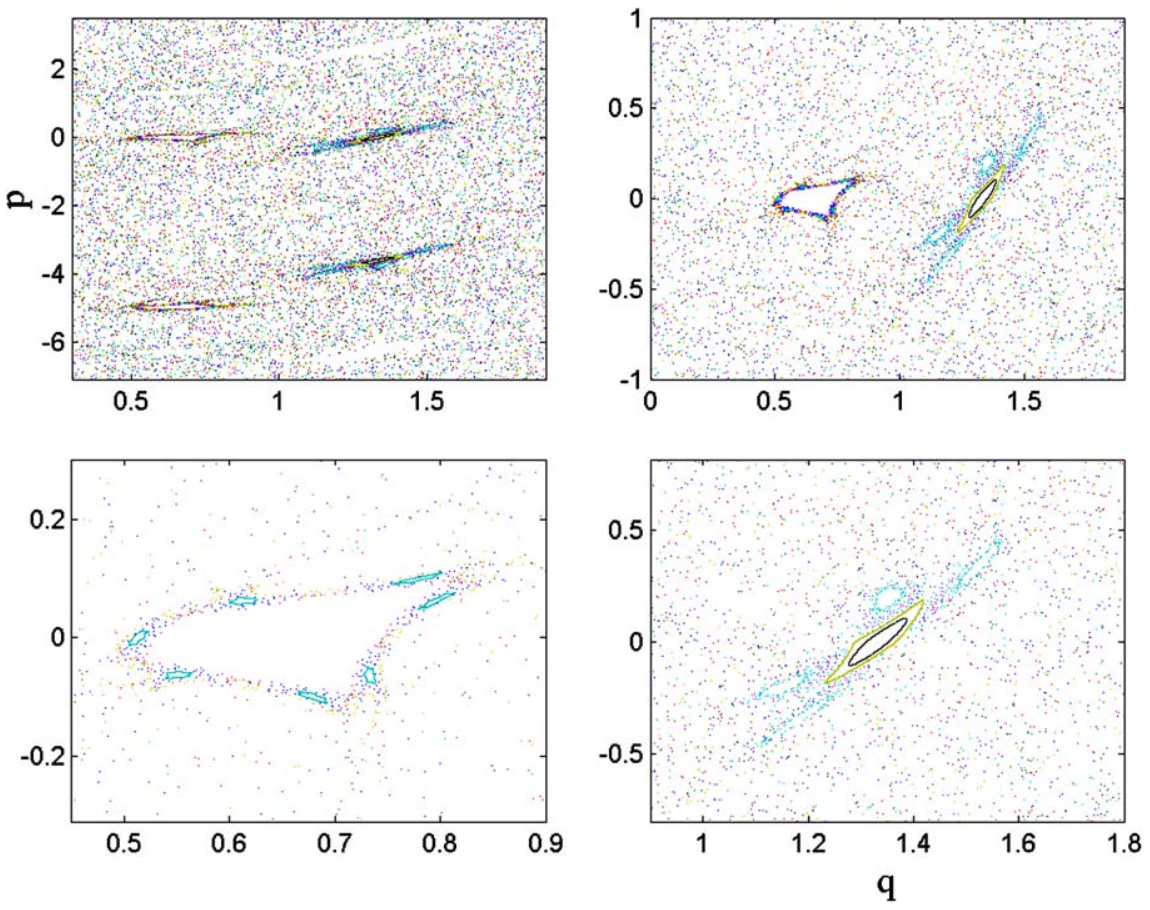

Fig. 4. The classical stroboscopic state space at $k=5$, each subplot is a zoom of different regions in the main plot in Fig. 1. 

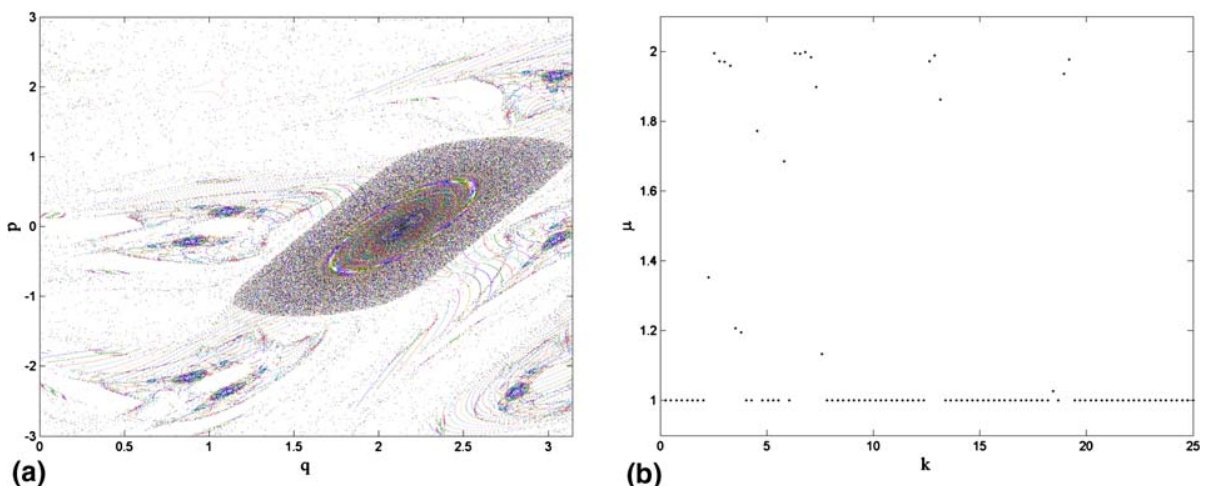

(b)

Fig. 5. A region of the accelerator mode in the classical stroboscopic state space at $k=2.5$ (a). The classical transport exponent $\mu$ versus perturbation strength $k(\mathrm{~b})$.

\section{Quantum dynamics}

The evolution operator of the quantum system is given by

$$
\hat{U}=\exp \left(\frac{-\mathrm{i} \hat{P}^{2} T}{4 \hbar}\right) \exp \left(\frac{-\mathrm{i} k \cos (q+\alpha)}{\hbar}\right) \exp \left(\frac{-\mathrm{i} \hat{P}^{2} T}{4 \hbar}\right) .
$$

The time development of a Floquet system can be studied by expanding the evolution operator in a suitable basis, i.e., the eigenstates of the unperturbed Hamiltonian $H_{0}$ :

$$
H_{0}\left|\psi_{n}\right\rangle=E_{n}\left|\psi_{n}\right\rangle
$$

where $E_{n}$ is the energy of the $n$th energy eigenvalue. $\left|\psi_{n}\right\rangle$ are the eigenfunctions of the Hamiltonian $H_{0}$ given by [22]

$$
\left\langle q \mid \psi_{n}\right\rangle=\sqrt{\frac{1}{a}} \sin \left(\frac{n \pi q}{2 a}\right), \quad E_{n}=\frac{\hbar^{2} n^{2} \pi^{2}}{8 m a^{2}}
$$

where $n=1,2,3 \ldots, N$. The evolution matrix $U$ is then calculated as

$$
U_{n m}=\left\langle\psi_{n}|U| \psi_{m}\right\rangle
$$

with the following analytical result [23]:

$$
\begin{aligned}
U_{n m}= & C \int_{0}^{\pi} \cos (\beta q) \mathrm{e}^{\mathrm{i} z \cos (q+\alpha)} \mathrm{d} q \\
= & C\left[\pi \mathrm{i}^{\beta} J_{\beta}(z) \cos (\beta \alpha)-4 \sum_{l=2,4}^{\infty} \mathrm{i}^{l} J_{l}(z) \sin (l \alpha)\left(\frac{l}{l^{2}-\beta^{2}}\right)\right] \text { for } \beta \text { odd } \\
& +C\left[\pi \mathrm{i}^{\beta} J_{\beta}(z) \cos (\beta \alpha)-4 \sum_{l=1,3}^{\infty} \mathrm{i}^{l} J_{l}(z) \sin (l \alpha)\left(\frac{l}{l^{2}-\beta^{2}}\right)\right] \quad \text { for } \beta \text { even, }
\end{aligned}
$$

where the constant $C=\frac{1}{\pi} \mathrm{e}^{-\frac{i \hbar h\left(n^{2}+m^{2}\right) T}{4}}$ and $\beta=n+m$ or $\beta=n-m$ and $J_{\beta}$ and $J_{l}$ are Bessel functions of order $\beta$ and $l$, respectively [24].

\subsection{Quantum energy and diffusion}

An arbitrary state of the system at time $t$ is given by

$$
|\Psi(t)\rangle=\sum_{n} A_{n}(t)\left|\psi_{n}\right\rangle .
$$

The iterates of the particle wavefunction $|\Psi(t)\rangle$ can be obtained via their expansion amplitudes, $A_{n}(t)$, to give the time evolution of any state of the system by matrix multiplication according to 


$$
A_{n}(t)=\sum_{m=1}^{N} U_{n m} A_{m}(0) .
$$

The energy of the kicked particle in an infinite potential well is given by a similar expression as for the kicked rotor [25]:

$$
E^{q m}(t)=\sum_{n=1}^{N} b_{n}(t) \hbar^{2} n^{2} / 2, \quad b_{n}(t)=\left|A_{n}(t)\right|^{2},
$$

where $b_{n}(t)$ is a probability distribution in momentum space after $t$ kicks of the perturbation where the initial amplitude was taken to be

$$
A_{n}(0)= \begin{cases}1, & n=1, \\ 0, & \text { otherwise. }\end{cases}
$$

The same quantum suppression of classical diffusion observed in the kicked rotor is observed in this system.

\subsection{Quantum diffusion coefficient $D_{q m}$}

The quantum diffusion coefficient $D_{\mathrm{qm}}$ was taken to be

$$
E(t)=D_{\mathrm{qm}} t
$$

and was calculated by a linear least squares fit of $E(t)$ to $t$ up to the saturation point and is shown in Fig. 6 .

The diffusion coefficient for $k<1$ is found to be effectively zero. The diffusion coefficient for $k>1$ was found to scale as $D_{\mathrm{qm}} \sim k^{1.3}$, for $1<k<10^{3}$. In the region $0<k<25$, the diffusion coefficient was found to scale as $D_{\mathrm{qm}} \sim k^{2} / 4.2$ shown in green. Also plotted in this figure are the predicted classical diffusion coefficient $D \sim k^{2} / 4$ (blue) and the classical analytic diffusion coefficient (red) defined for the standard map in [19,26,27]. The quantum diffusion coefficient mimics the classical analytical value in that numerous oscillations are present but these occur at different $k$ values than for the classical analytical result. In order to establish an analytical quantum diffusion expression for the kicked rotor,
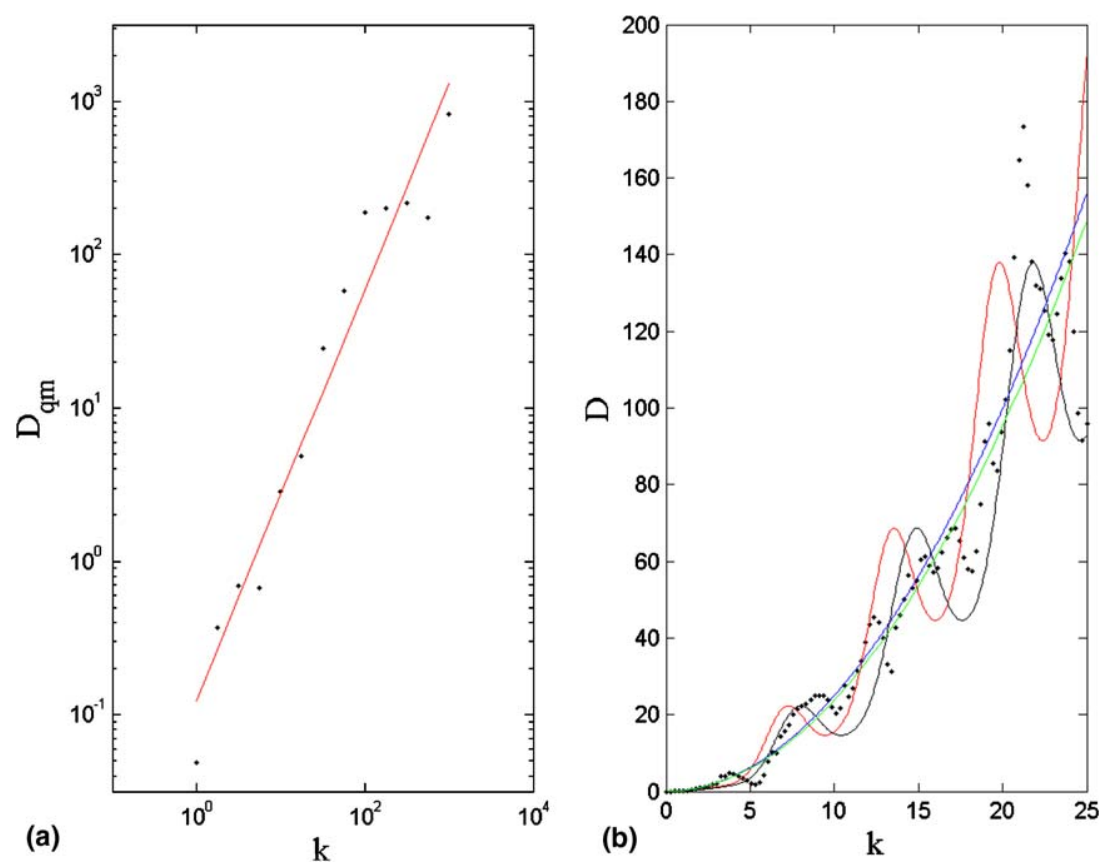

Fig. 6. (a) The quantum diffusion coefficient $D_{\mathrm{qm}}$ versus $k$ for $1<k<10^{3}$. (b) The diffusion coefficient (black dots) for $0<k<25$, fitted diffusion coefficient (green), quantum analytical diffusion coefficient (black), predicted classical diffusion coefficient (blue) and classical analytic diffusion coefficient (red) superimposed. (For interpretation of colour in this figure reader is referred to web version of this article.) 
Shepelyansky calculated the first few quantum correlations, and found they had the same form as the classical correlations upon the substitution $[28,29]$,

$$
k \rightarrow\left[\frac{\sin k^{*} / 2}{k^{*} / 2}\right] k .
$$

This approach was applied successfully in [19], where $k^{*} \approx 2.08$. The analytical expression for the quantum diffusion coefficient is also presented in Fig. 6, for a value of $k^{*}=1.5$. These tentative results are seen to approximate the numerical data in the range shown.

\section{The quantum 'quasi' phase space}

\subsection{Derivation of the Wigner distribution function}

A general expression for the superposition of basis states of the kicked particle in an infinite potential well is given by

$$
\Psi(x)=\frac{1}{\sqrt{a}} \sum_{n=1}^{\infty} A_{n} \sin \left(\frac{n \pi x}{2 a}\right) .
$$

To determine the initial Wigner distribution function we use the definition of the Wigner distribution function for a 1-dimensional system, given in general as [30,31]

$$
F^{\mathrm{W}}(q, p, t)=\frac{1}{(\pi \hbar)} \int_{-\infty}^{\infty} \mathrm{d} x \mathrm{e}^{-2 i p x / \hbar} \Psi^{*}(q-x, t) \Psi(q+x, t),
$$

and replace $a$ with $\pi / 2$ in $\Psi(x)$

$$
\Psi(x)=\frac{1}{2 \mathrm{i}} \sqrt{\frac{2}{\pi}} \sum_{n=1}^{\infty} A_{n}\left(\mathrm{e}^{\mathrm{i} n x}-\mathrm{e}^{-\mathrm{i} n x}\right) .
$$

Therefore

$$
\begin{aligned}
& \Psi(q+x)=\frac{1}{2 \mathrm{i}} \sqrt{\frac{2}{\pi}} \sum_{n=1}^{\infty} A_{n}\left(\mathrm{e}^{\mathrm{i} n(q+x)}-\mathrm{e}^{-\mathrm{i} i(q+x)}\right), \\
& \Psi^{*}(q-x)=\frac{1}{2 \mathrm{i}} \sqrt{\frac{2}{\pi}} \sum_{m=1}^{\infty} A_{m}^{*}\left(\mathrm{e}^{-\mathrm{i} m(q-x)}-\mathrm{e}^{\mathrm{i} m(q-x)}\right) .
\end{aligned}
$$

Substituting Eqs. (15) and (16) into Eq. (13), the Wigner distribution function can now be written as

$$
F^{\mathrm{W}}\left(q, p_{k}\right)=\frac{1}{2 \pi^{2} \hbar} \int \sum_{m=1}^{\infty} \sum_{n=1}^{\infty} A_{m}^{*} A_{n}\left(\mathrm{e}^{-\mathrm{i} m(q-x)}-\mathrm{e}^{\mathrm{i} m(q-x)}\right)\left(\mathrm{e}^{\mathrm{i} n(q+x)}-\mathrm{e}^{-\mathrm{i} n(q+x)}\right) \mathrm{e}^{-2 \mathrm{i} k x} \mathrm{~d} x,
$$

where $k=p / \hbar$. Through further manipulation, the Wigner distribution reduces to the following:

$$
\begin{aligned}
F^{\mathrm{W}}\left(q, p_{k}\right)= & \frac{1}{2 \pi^{2} \hbar} \sum_{m=1}^{\infty} \sum_{n=1}^{\infty} A_{m}^{*} A_{n}\left[\mathrm{e}^{-\mathrm{i}(m-n) q} \int \mathrm{e}^{\mathrm{i}(m+n-2 k) x} \mathrm{~d} x-\mathrm{e}^{-\mathrm{i}(m+n) q} \int \mathrm{e}^{\mathrm{i}(m-n-2 k) x} \mathrm{~d} x\right. \\
& \left.-\mathrm{e}^{-\mathrm{i}(m+n) q} \int \mathrm{e}^{-\mathrm{i}(m-n+2 k) x} \mathrm{~d} x+\mathrm{e}^{\mathrm{i}(m-n) q} \int \mathrm{e}^{-\mathrm{i}(m+n+2 k) x} \mathrm{~d} x\right] .
\end{aligned}
$$

The integration is performed in Appendix A and the resulting analytical expression for the Wigner distribution function is presented there for different integration limits.

\subsection{Derivation of the Husimi distribution function}

The Husimi distribution function is a Gaussian smoothed Wigner distribution function. To determine the initial Husimi distribution function we use the definition of the Husimi distribution function $[32,33,31]$

$$
F^{\mathrm{H}}(q, p, t)=\frac{1}{2 \pi \hbar}\left|\int \mathrm{d} x \varrho_{q p}^{*}(x) \Psi(x, t)\right|^{2}
$$


and

$$
\left\langle x \mid \varrho_{q p}\right\rangle \equiv \varrho_{q p}(x)=\left(\frac{s}{\pi \hbar}\right)^{\frac{1}{4}} \mathrm{e}^{-s(x-q)^{2} / 2 \hbar} \mathrm{e}^{\mathrm{i} p x / \hbar},
$$

where $\left|\varrho_{q p}\right\rangle$ represents the wave function for a minimum uncertainty squeezed Gaussian wave packet peaked at $(q, p)$. $s$ is the squeezing parameter which determines the relative resolution in the $q$ space (large $s$ ) versus the $p$ space (small $s$ ) [31]. Using $\Psi_{n}(x)$ from Eq. (12), the Husimi distribution function becomes

$$
F^{\mathrm{H}}(q, p)=\frac{1}{2 \pi \hbar} \sqrt{\frac{s}{\pi \hbar}}\left|\int_{0}^{2 a} \mathrm{~d} x \sqrt{\frac{1}{a}} \sum_{n=1}^{\infty} A_{n} \sin \left(\frac{n \pi x}{2 a}\right) \mathrm{e}^{-s(x-q)^{2} / 2 \hbar} \mathrm{e}^{\mathrm{i} p x / \hbar}\right|^{2} .
$$

Using Eq. (14), the Husimi distribution function becomes

$$
F^{\mathrm{H}}(q, p)=\frac{1}{8 \pi \hbar a} \sqrt{\frac{s}{\pi \hbar}}\left|\sum_{n=1}^{\infty} A_{n} \int_{0}^{2 a}\left(\mathrm{e}^{-s(x-q)^{2} / 2 \hbar} \mathrm{e}^{-\mathrm{i} p x / \hbar}\right)\left(\mathrm{e}^{\mathrm{i} n \pi x}-\mathrm{e}^{-\frac{\mathrm{i} n \pi x}{2 a}}\right) \mathrm{d} x\right|^{2} .
$$

The integration is performed in Appendix B and the resulting analytical expression for the Husimi distribution function is given by

$$
F^{\mathrm{H}}(q, p)=\frac{1}{16 a s \sqrt{\pi \hbar}}\left|\sum_{n=1}^{\infty} A_{n}\left\{\mathrm{e}^{-\mathrm{i}\left(\frac{p}{h} \frac{n \pi}{2 a}\right) q} \mathrm{e}^{-p_{1}^{2}}\left[\operatorname{erf}\left(-q_{1}+\mathrm{i} p_{1}\right)-\operatorname{erf}\left(-q_{2}+\mathrm{i} p_{1}\right)\right]-\mathrm{e}^{-\mathrm{i}\left(\frac{p}{h} \frac{n \pi}{2 a}\right) q} \mathrm{e}^{-p_{2}^{2}}\left[\operatorname{erf}\left(-q_{1}+\mathrm{i} p_{2}\right)-\operatorname{erf}\left(-q_{2}+\mathrm{i} p_{2}\right)\right]\right\}\right|^{2},
$$

where $\operatorname{erf}(z)$ denotes the error function of complex argument $z$, and

$$
\begin{aligned}
q_{1} & =\sqrt{\frac{s}{2 \hbar}}(q-2 a), & q_{2} & =\sqrt{\frac{s}{2 \hbar}} q, \\
p_{1} & =\sqrt{\frac{\hbar}{2 s}}\left(\frac{p}{\hbar}-\frac{n \pi}{2 a}\right), & p_{2} & =\sqrt{\frac{\hbar}{2 s}}\left(\frac{p}{\hbar}+\frac{n \pi}{2 a}\right) .
\end{aligned}
$$

The analytical solutions of the Wigner and Husimi distribution functions for the periodically kicked particle in an infinite potential well have been numerically verified in the present study using a fast Fourier transform method suggested in [34].

In [31], it was seen that the structure of the 10th eigenstate of a particle in an infinite potential well is very regular since the state is highly localized. Fig. 7 shows the Wigner distribution function for a slightly higher state, the 13th Floquet state, when a perturbation of $k=5$ is applied to the system. The phase space distribution has become distorted and it is no longer possible to see the detailed peak structure along the $q$ axis.

Also presented in this figure are the Husimi distribution functions for various $s$. As expected, for small $s$ remnants of the $p= \pm 13 \hbar$ structure in the $p$ axis is evident. However for larger $s, s \geqslant 80$, only two main peaks are visible along the $q$ axis. The first (and main peak) occurs at $q \sim 0.57$, which corresponds classically to the hyperbolic fixed point. The second peak occurs at $q \sim 2.14$, which is the position of the elliptic fixed point. This example illustrates the superiority of the Husimi distribution function in its ability to pick out the classical correspondence of the quantum system, which was hidden by the complicated structure of the Wigner distribution function. For even a perturbed system we are able to use the quasi-probability distribution functions to investigate the classical-quantum correspondence.

\subsection{Quasi-probability distributions of the Floquet states}

To investigate the classical-quantum correspondence further, we use as our amplitude, $A_{n}$, the Floquet states of the system, as they may be regarded as a quantum analog of the periodic orbits [31]. The time evolution of the system depends on whether the Floquet states have distributions near the elliptic fixed points or the hyperbolic fixed points [35]. The Floquet states were sorted according to their leading eigenbasis component. Three Floquet states were chosen, of high enough $n$ to be considered within the semiclassical approximation, but low enough $n$ so that the Wigner distribution function is not too greatly affected by high oscillations [31]. An intermediate value of $s=80$ was chosen for the calculation of the Husimi distribution function.

From Figs. 8 and 9, for $k=0.1$ it can be seen that $\psi_{19}$ has a probability distribution shifted slightly towards the hyperbolic fixed point, $\psi_{25}$ has a probability distribution shifted slightly towards the elliptic fixed point and $\psi_{22}$ has an equiprobability distribution to be localized at either fixed point. The probability amplitudes associated with these 

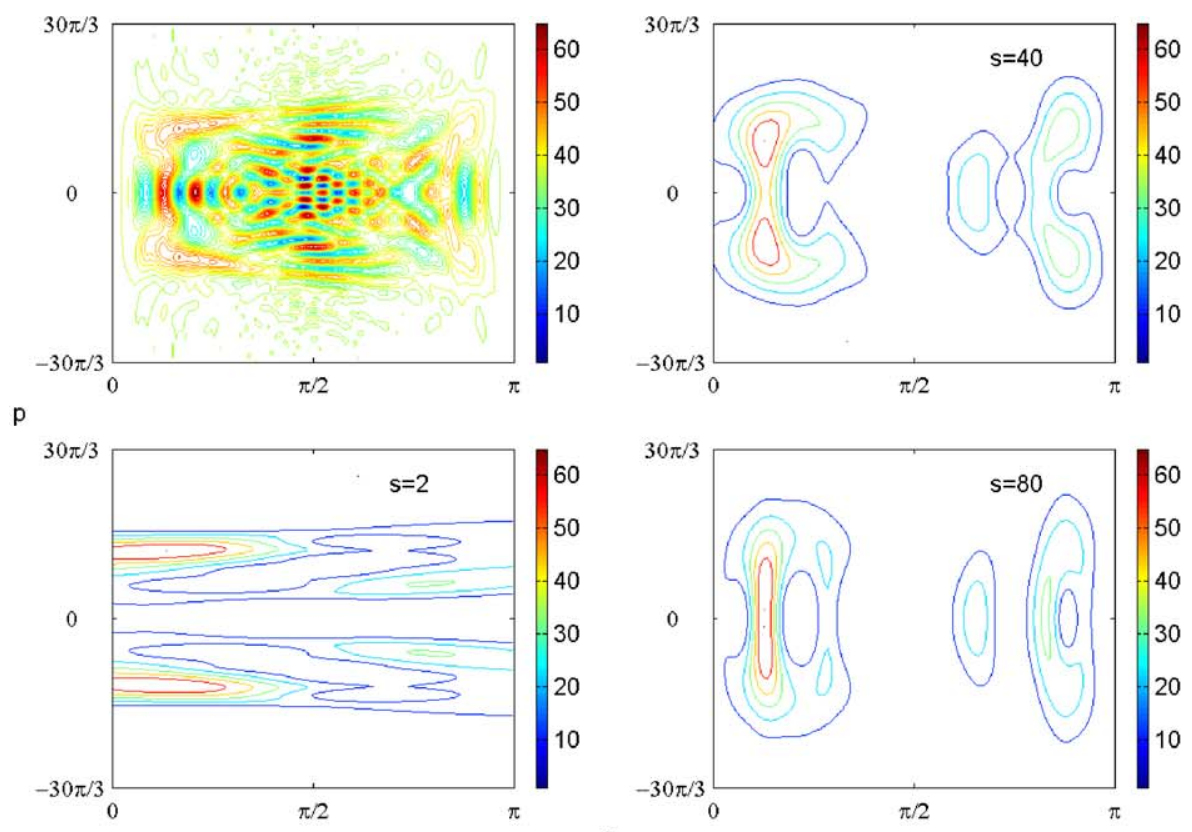

(a)
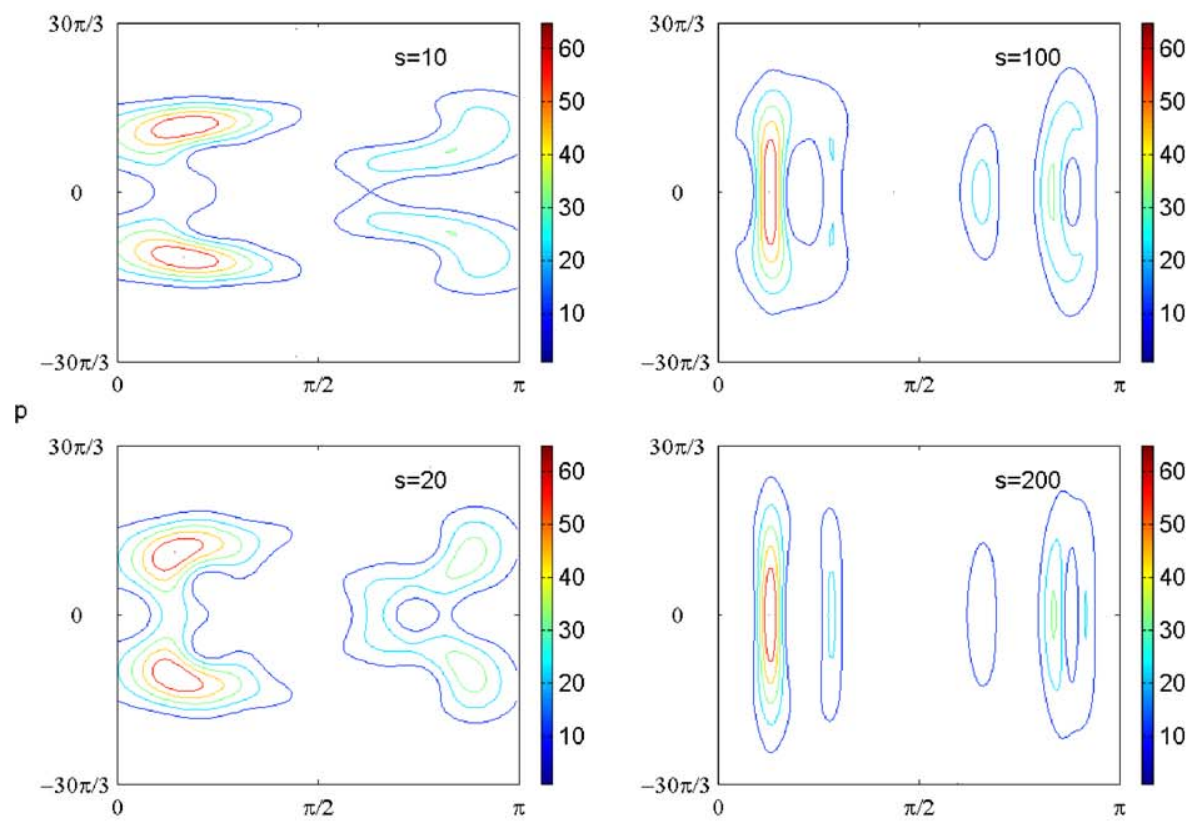

(b)

Fig. 7. The Wigner (upper left) and Husimi distribution functions of the 13th Floquet state, of the perturbed system $(k=5)$, for each of the squeezing parameters $(s)$ shown.

states are shown in Fig. 10 and on a logarithmic scale in Fig. 11. For $k=1$, the system is highly localized at $n=22$ (for the unshifted state $\psi_{22}$ ). However for $\psi_{19}$ apart from the main peak at $n=19$, a higher lying level contributes a second high amplitude peak, that may be associated with the shift of the probability towards the hyperbolic fixed point. This interpretation is supported by the presence of a lower-lying peak, as well as the main $n=25$ peak, in the case of $\psi_{25}$, which may be associated with the shift of the probability towards the elliptic fixed point for $\psi_{25}$. 

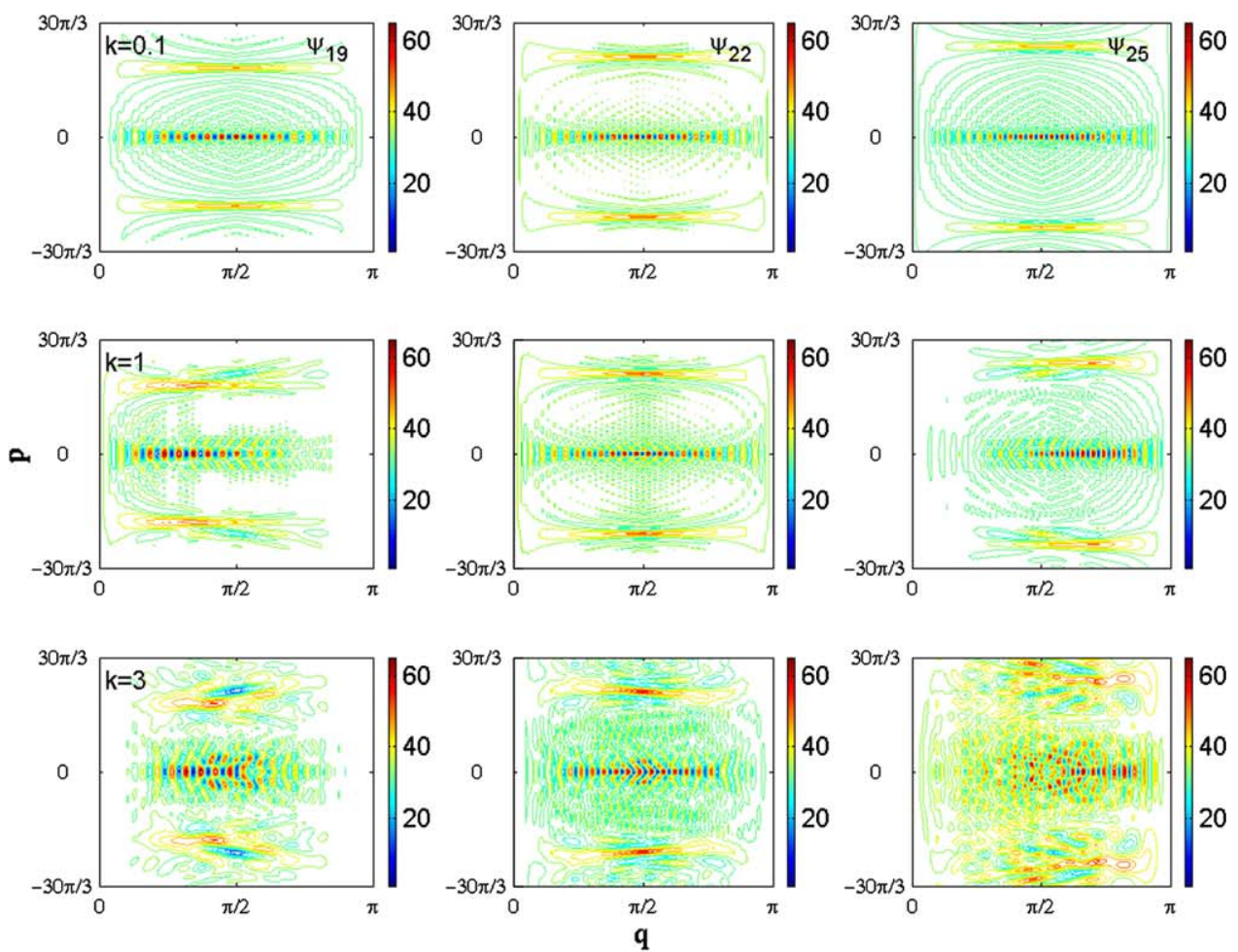

(a)
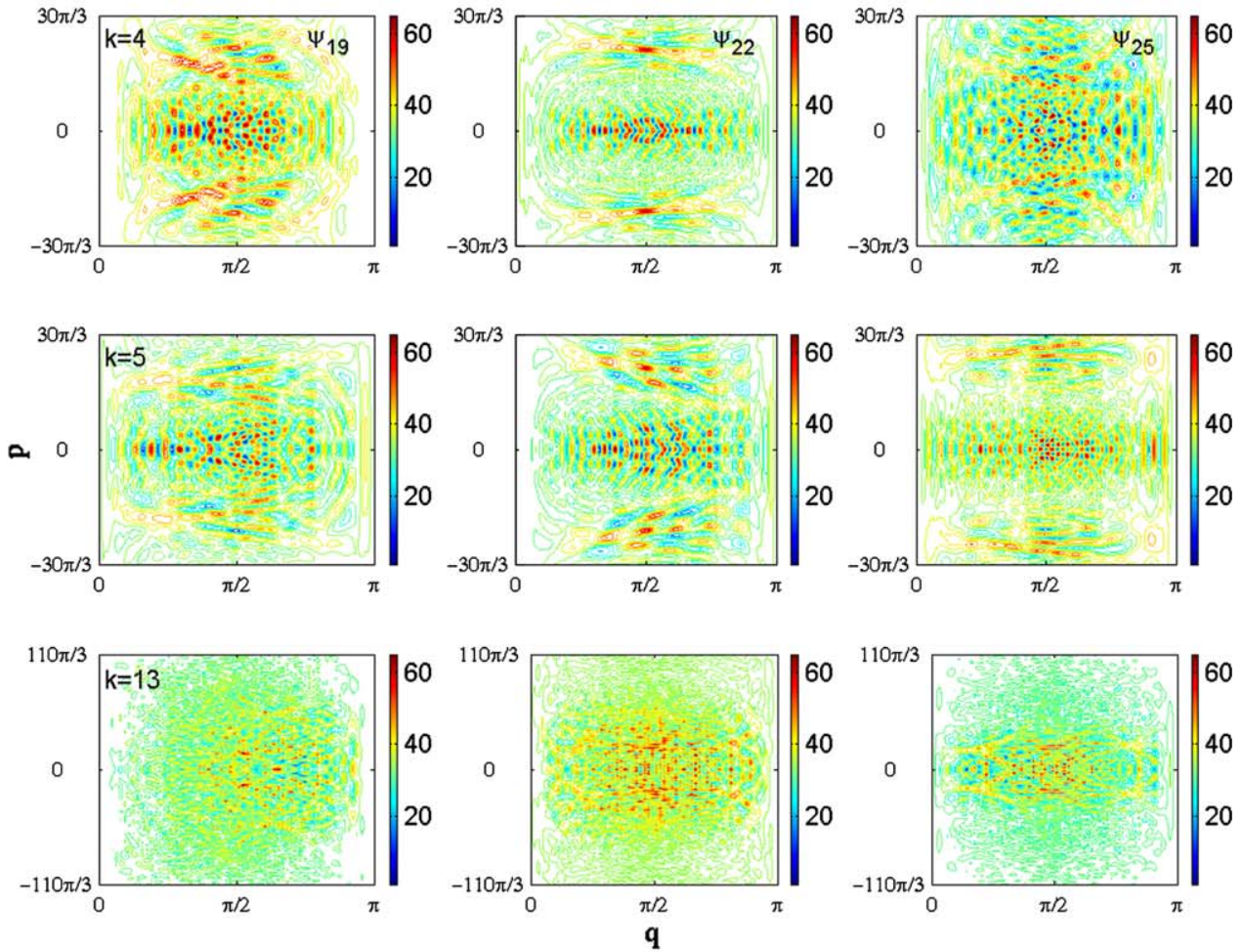

(b)

Fig. 8. The Wigner distribution functions of various Floquet states of the perturbed system, for each of the kick strengths shown 

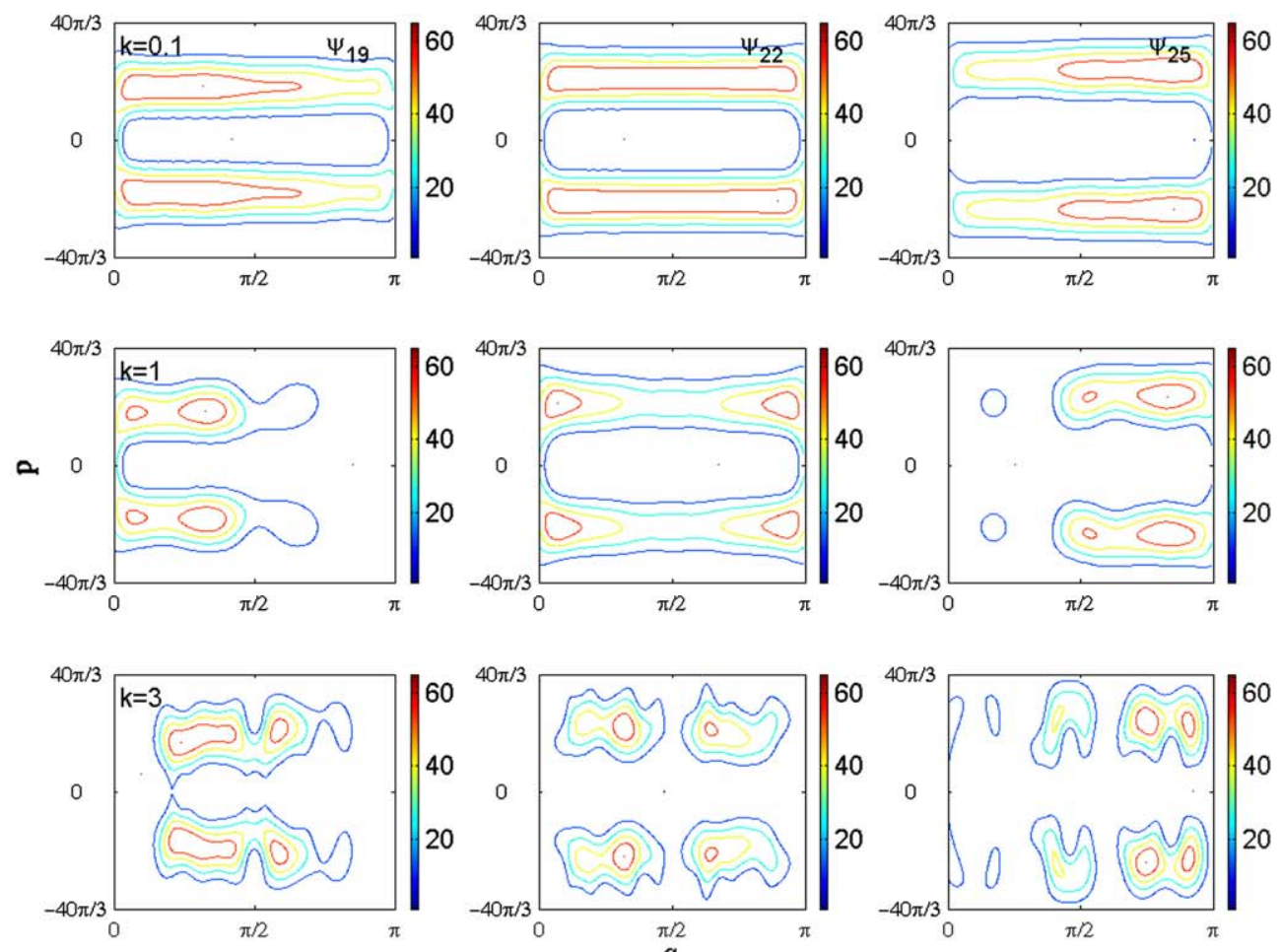

(a)
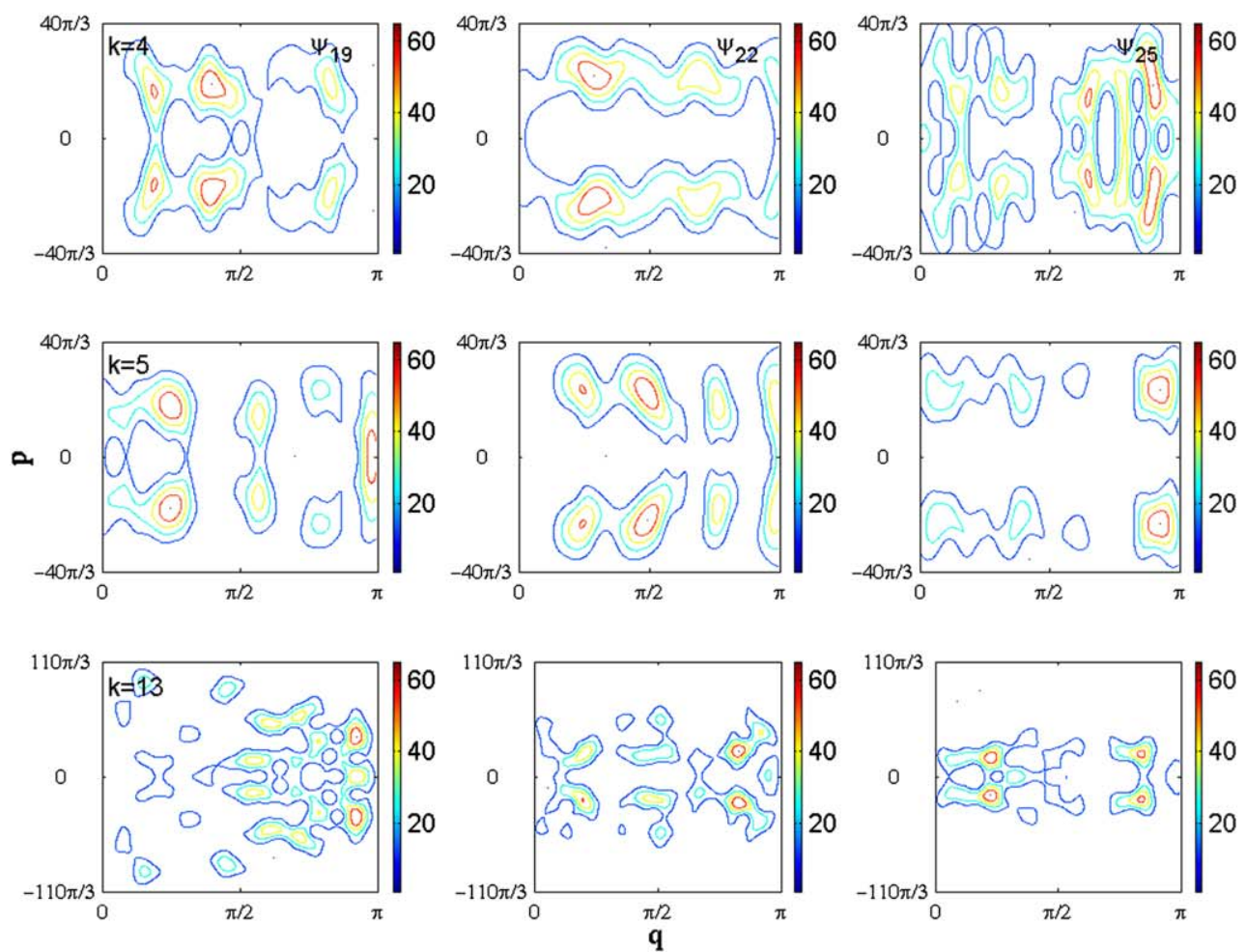

(b)

Fig. 9. The Husimi distribution functions of various Floquet states of the perturbed system, for each of the kick strengths shown. 

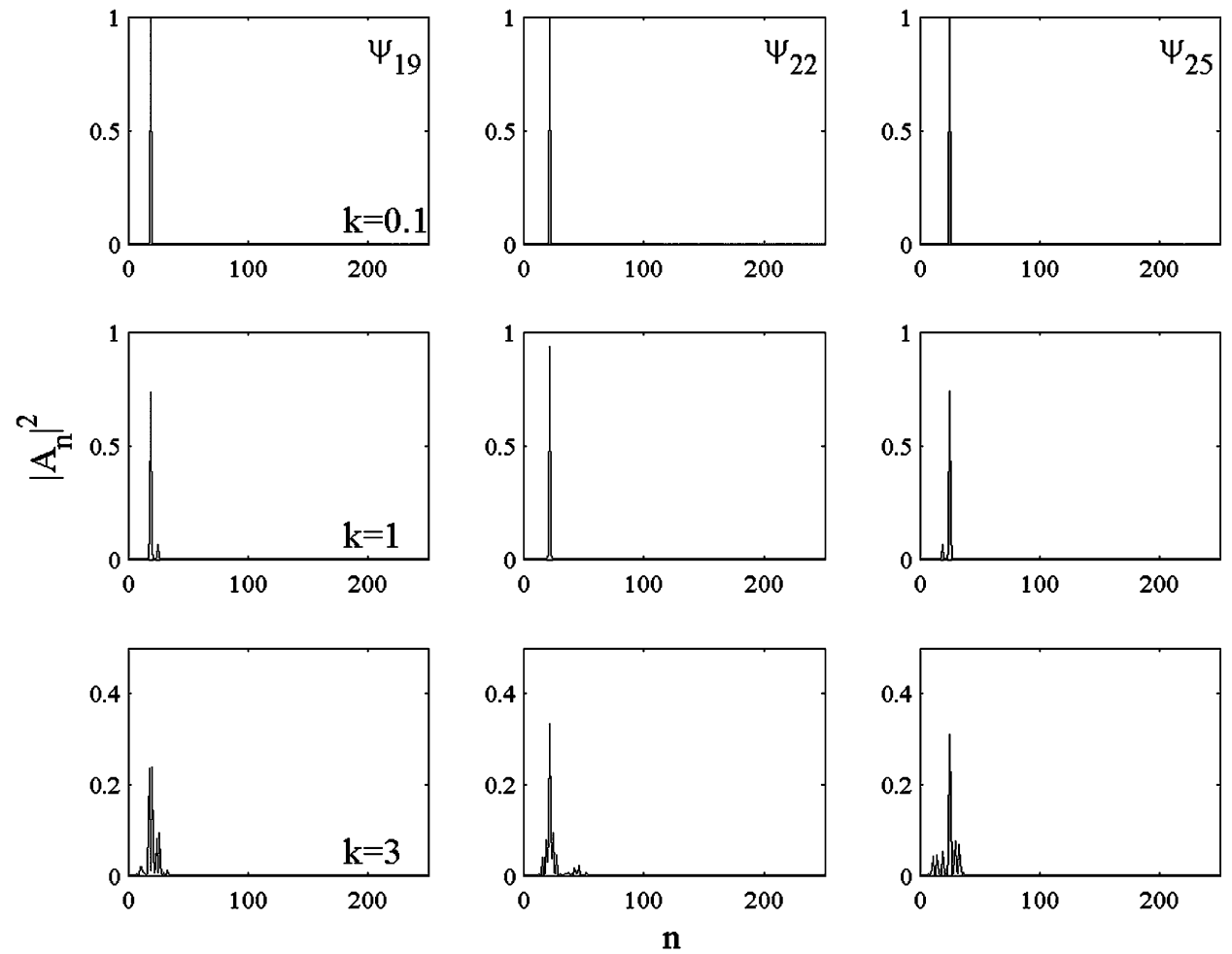

(a)
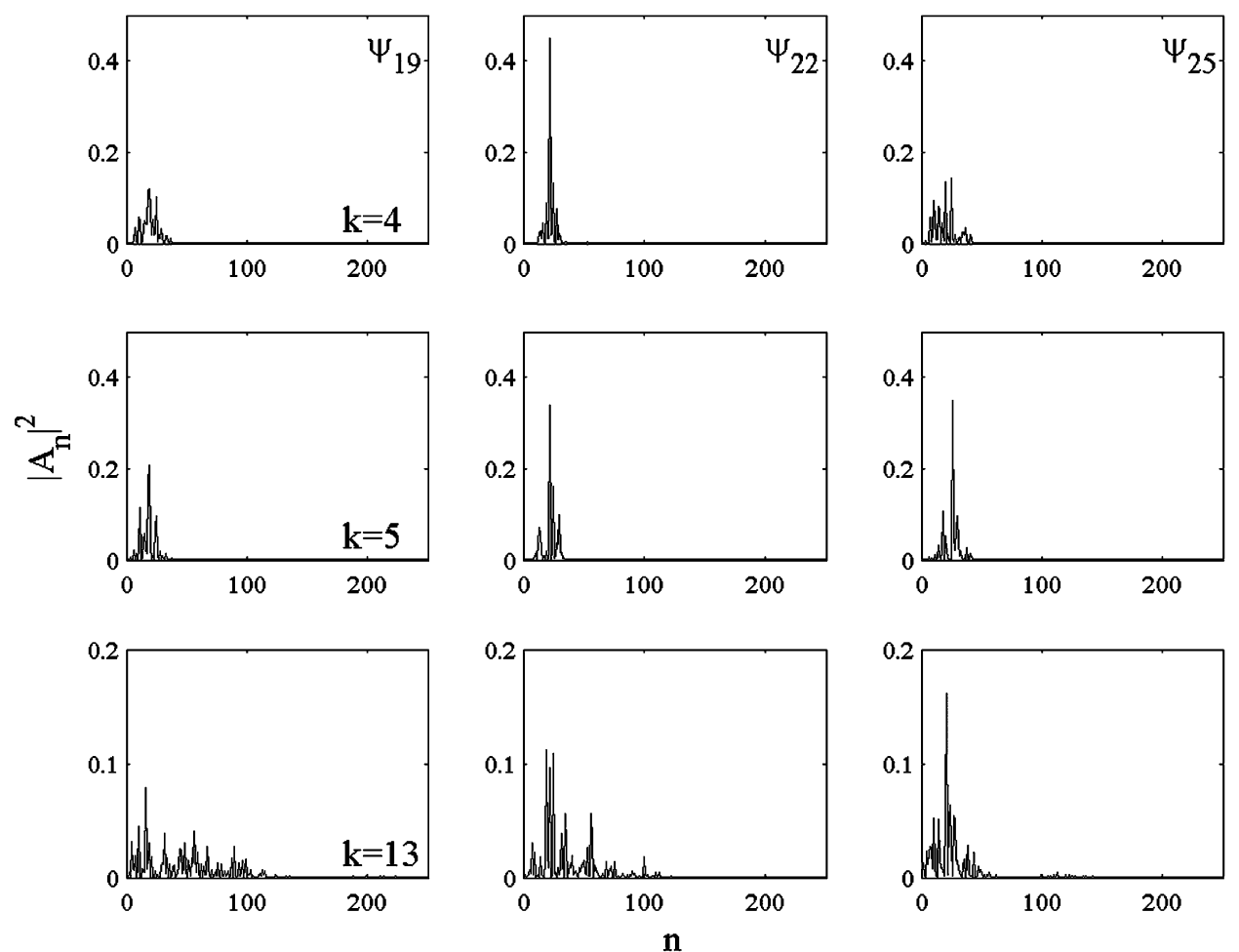

(b)

Fig. 10. The $\mid$ amplitude $\left.\right|^{2}$ of various Floquet states of the perturbed system, for each of the kick strengths shown. 

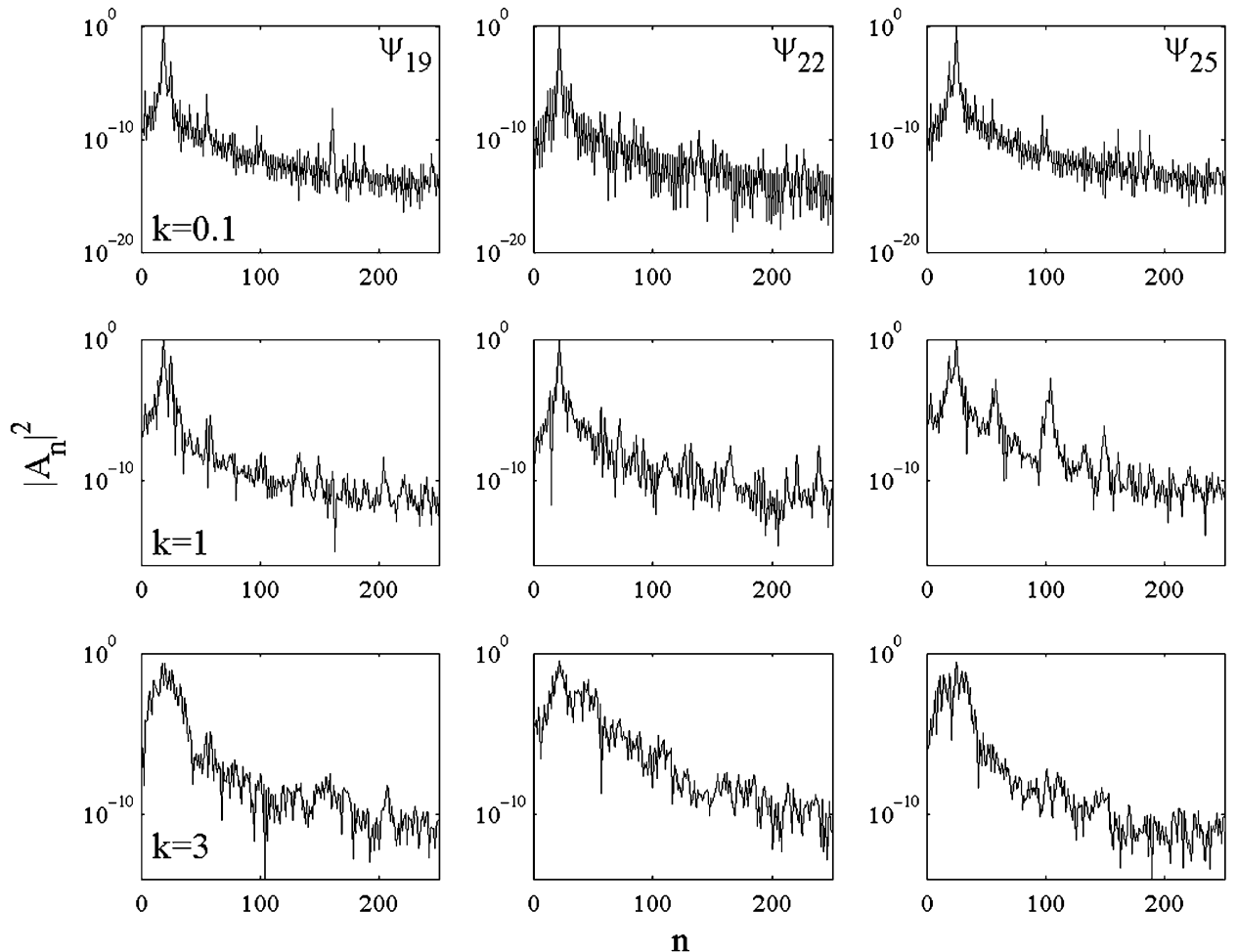

(a)
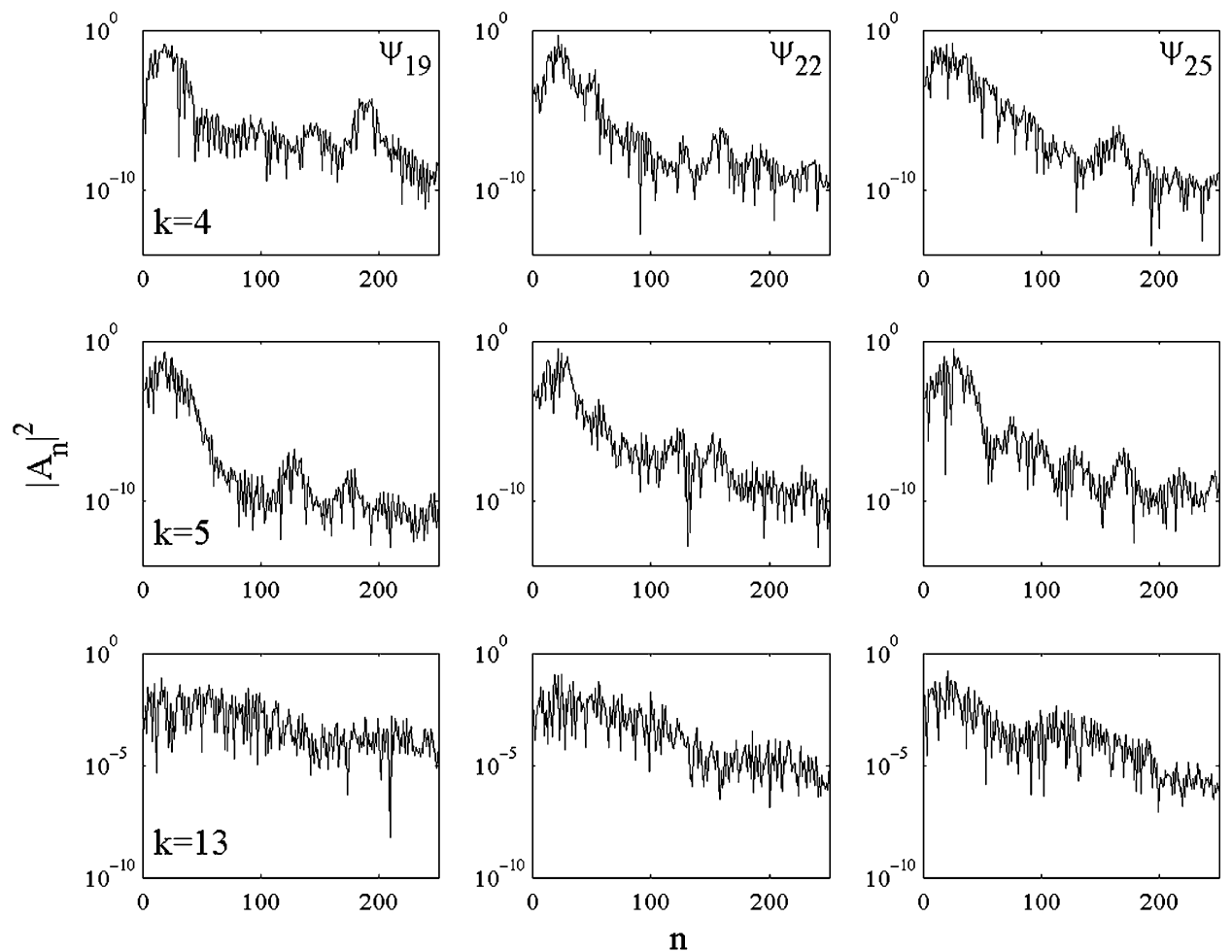

(b)

Fig. 11. The $\mid$ amplitude $\left.\right|^{2}$ on a logarithmic scale of various Floquet states of the perturbed system, for each of the kick strengths shown. 
For $k=3$ some deformations of the Floquet states are observed and reflected in their Wigner and Husimi representations. This is to be expected since we are close to the classical instability border $(k=4)$ and the stronger the local instability, the greater the deformation of phase space. This is also reflected in the spread of the probability amplitudes. The probability distribution for $\psi_{19}$ is seen to shift slightly towards the elliptic fixed point, owing to the fact that the spread in the amplitudes is towards lower $n$. The opposite is observed for $\psi_{25}$, where a spread in the amplitudes towards higher $n$ results in a shift of the probability distribution towards the hyperbolic fixed point. It is interesting to note that the probability distribution associated with $\psi_{22}$ remains quite uniform between the elliptic and hyperbolic fixed points, with the probability amplitude remaining highly localized around $n=22$, with small amounts of equal spread to higher and lower states.

At $k=4$ the probability distributions for both states $\psi_{19}$ and $\psi_{25}$ are well spread over phase space. The spread is also seen in the probability amplitudes for $\psi_{19}$ and $\psi_{25}$, which are quite extended. $\psi_{22}$ still remains relatively localized with small and equal contributions from higher and lower $n$ not being strong enough to significantly influence the probability distribution towards the elliptic or hyperbolic fixed points.

Unexpectedly for $k=5$ the Floquet states becomes slightly more localized again. This is reflected in the diffusion coefficient where a dip is seen at $k=5$. At $k=13$, the probability amplitude of $\psi_{19}$ (located near the hyperbolic fixed point) is extended and a large spread of the Floquet state in momentum is observed. $\psi_{22}$ is seen to become more delocalized, whereas $\psi_{25}$, which for small $k$ has a probability distribution to be located near the elliptic fixed point remains most localized.

\section{Conclusion}

The aim of the present study was to investigate the classical-quantum correspondence for the system of a periodically kicked particle in an infinite potential well. Classically a stochastic web exists containing many chaotic trajectories for any $k>0$. As $k$ increases, the stochastic layer becomes wider and eventually appears to cover the whole of phase space. However the existence of stable islands were observed for $k=5$. A diffusion study revealed that in the region $0<k<25$, for some values of $k$, the energy was found to grow quadratically with time. In analogy with the kicked rotor, accelerator modes were found to exist in the system at $k=\pi, 2 \eta \pi, \ldots$ where $\eta=1, \ldots, N$. In order to establish a correspondence with the classical system, the quantum evolution of the amplitudes and energy was investigated. It was found that the quantum diffusion scales on average as $D_{\mathrm{qm}} \propto k^{2} / 4.2$. The quantum diffusion coefficient was found to mimic the classical diffusion coefficient by rescaling $k$.

The classical-quantum correspondence was further investigated with the use of quantum quasi-probability distribution functions and the Floquet states of the system. Analytical solutions for both the Wigner and Husimi distribution functions were presented. In particular it was found that the Husimi distribution function gives a more informative description of the system. Contributions from high-lying levels (relative to the most dominant basis state) of the unperturbed Hamiltonian gives rise to probability distributions located close to the hyperbolic fixed point, whereas contributions from low-lying levels give rise to probability distributions close to the elliptic fixed point of the classical map. The change in the quasi-phase space of these Floquet states as the perturbation is increased was investigated. Remnants of the bifurcation in the classical system at $k=4$ were observed. The system was seen to become more ergodic for the Floquet state located near the hyperbolic fixed point than near the elliptic fixed point for $k=13$.

\section{Acknowledgement}

This work was supported by the Irish Science and Technology Agency Enterprise Ireland under research grant SC/ 99/206.

\section{Appendix A. Derivation of the Wigner distribution function}

Boundary conditions of $\Psi=0$ at $q=0$ and $q=\pi$, leads to three ranges of integration:

(1) Limits for $0<q<\frac{\pi}{2}$

$$
\begin{aligned}
\int_{-q}^{q} \mathrm{e}^{\mathrm{i} \gamma x} \mathrm{~d} x & =\frac{\mathrm{e}^{\mathrm{i} \gamma q}-\mathrm{e}^{-\mathrm{i} \gamma q}}{\mathrm{i} \gamma} \text { for } \gamma \neq 0 \\
& =2 q \text { for } \gamma=0 .
\end{aligned}
$$


(2) Limits for $q=\frac{\pi}{2}$

$$
\begin{aligned}
\int_{-\pi / 2}^{\pi / 2} \mathrm{e}^{\mathrm{i} \gamma x} \mathrm{~d} x & =\frac{\mathrm{e}^{\mathrm{i} \gamma \frac{\pi}{2}}-\mathrm{e}^{-\mathrm{i} \gamma \frac{\pi}{2}}}{\mathrm{i} \gamma} \quad \text { for } \gamma \neq 0 \\
& =\pi \quad \text { for } \gamma=0 .
\end{aligned}
$$

(3) Limits for $\frac{\pi}{2}<q<\pi$

$$
\begin{aligned}
\int_{-(\pi-q)}^{(\pi-q)} \mathrm{e}^{\mathrm{i} \gamma x} \mathrm{~d} x & =\frac{\mathrm{e}^{\mathrm{i} \gamma(\pi-q)}-\mathrm{e}^{-\mathrm{i} \gamma(\pi-q)}}{\mathrm{i} \gamma} \text { for } \gamma \neq 0 \\
& =2(\pi-q) \text { for } \gamma=0 .
\end{aligned}
$$

The Wigner distribution function for the particle in an infinite potential well can now be calculated for each value of $\gamma$, $(\gamma=m+n-2 k, \gamma=m-n-2 k, \gamma=m-n+2 k, \gamma=m+n+2 k)$. The following is the resulting analytical expression for the Wigner distribution function of a kicked particle in an infinite potential well with boundaries at 0 and $\pi$ :

(1) For $0<q<\frac{\pi}{2}$

$$
\begin{aligned}
F^{\mathrm{W}}\left(q, p_{k}\right)= & \frac{1}{2 \pi^{2} \hbar} \sum_{m=1}^{\infty} \sum_{n=1}^{\infty} A_{m}^{*} A_{n} \\
& \times \mathrm{e}^{-\mathrm{i}(m-n) q} \begin{cases}\frac{\mathrm{e}^{\mathrm{i}(m+n-2 k) q}-\mathrm{e}^{-\mathrm{i}(m+n-2 k) q}}{\mathrm{i}(m+n-2 k)} & \text { if } m+n-2 k \neq 0 \\
2 q & \text { if } m+n-2 k=0\end{cases} \\
& -\mathrm{e}^{\mathrm{i}(m+n) q} \begin{cases}\frac{\mathrm{e}^{\mathrm{i}(m-n-2 k) q}-\mathrm{e}^{-\mathrm{i}(m-n-2 k) q}}{\mathrm{i}(m-n-2 k)} & \text { if } m-n-2 k \neq 0 \\
2 q & \text { if } m-n-2 k=0\end{cases} \\
& +\mathrm{e}^{\mathrm{i}(m-n) q} \begin{cases}\frac{\mathrm{e}^{\mathrm{i}(m-n+2 k) q}-\mathrm{e}^{-\mathrm{i}(m-n+2 k) q}}{\mathrm{i}(m-n+2 k)} & \text { if } m-n+2 k \neq 0 \\
2 q & \text { if } m-n+2 k=0 \\
2 q & \text { if } m+n+2 k \neq 0\end{cases}
\end{aligned}
$$

(2) For $q=\frac{\pi}{2}$

$$
\begin{aligned}
& F^{\mathrm{W}}\left(q, p_{k}\right)=\frac{1}{2 \pi^{2} \hbar} \sum_{m=1}^{\infty} \sum_{n=1}^{\infty} A_{m}^{*} A_{n} \\
& \times \mathrm{e}^{-\mathrm{i}(m-n) q} \begin{cases}\frac{\mathrm{e}^{\mathrm{i}(m+n-2 k) \frac{\pi}{2}}-\mathrm{e}^{-\mathrm{i}(m+n-2 k) \frac{\pi}{2}}}{\mathrm{i}(m+n-2 k)} & \text { if } m+n-2 k \neq 0 \\
\pi & \text { if } m+n-2 k=0\end{cases} \\
& -\mathrm{e}^{\mathrm{i}(m+n) q} \begin{cases}\frac{\mathrm{e}^{\mathrm{i}(m-n-2 k) \frac{\pi}{2}}-\mathrm{e}^{-\mathrm{i}(m-n-2 k) \frac{\pi}{2}}}{\mathrm{i}(m-n-2 k)} & \text { if } m-n-2 k \neq 0 \\
\pi & \text { if } m-n-2 k=0\end{cases} \\
& -\mathrm{e}^{-\mathrm{i}(m+n) q} \begin{cases}\frac{\mathrm{e}^{\mathrm{i}(m-n+2 k) \frac{\pi}{2}}-\mathrm{e}^{-\mathrm{i}(m-n+2 k) \frac{\pi}{2}}}{\mathrm{i}(m-n+2 k)} & \text { if } m-n+2 k \neq 0 \\
\pi & \text { if } m-n+2 k=0\end{cases} \\
& +\mathrm{e}^{\mathrm{i}(m-n) q} \begin{cases}\frac{\mathrm{e}^{\mathrm{i}(m+n+2 k) \frac{\pi}{2}}-\mathrm{e}^{-\mathrm{i}(m+n+2 k) \frac{\pi}{2}}}{\mathrm{i}(m+n+2 k)} & \text { if } m+n+2 k \neq 0 \\
\pi & \text { if } m+n+2 k=0 .\end{cases}
\end{aligned}
$$


(3) For $\frac{\pi}{2}<q<\pi$,

$$
\begin{aligned}
& F^{\mathrm{W}}\left(q, p_{k}\right)=\frac{1}{2 \pi^{2} \hbar} \sum_{m=1}^{\infty} \sum_{n=1}^{\infty} A_{m}^{*} A_{n} \\
& \times \mathrm{e}^{-\mathrm{i}(m-n) q} \begin{cases}\frac{\mathrm{e}^{\mathrm{i}(m+n-2 k)(\pi-q)}-\mathrm{e}^{-\mathrm{i}(m+n-2 k)(\pi-q)}}{\mathrm{i}(m+n-2 k)} & \text { if } m+n-2 k \neq 0 \\
2(\pi-q) & \text { if } m+n-2 k=0\end{cases} \\
& -\mathrm{e}^{\mathrm{i}(m+n) q} \begin{cases}\frac{\mathrm{e}^{\mathrm{i}(m-n-2 k)(\pi-q)}-\mathrm{e}^{-\mathrm{i}(m-n-2 k)(\pi-q)}}{\mathrm{i}(m-n-2 k)} & \text { if } m-n-2 k \neq 0 \\
2(\pi-q) & \text { if } m-n-2 k=0\end{cases} \\
& -\mathrm{e}^{-\mathrm{i}(m+n) q} \begin{cases}\frac{\mathrm{e}^{\mathrm{i}(m-n+2 k)(\pi-q)}-\mathrm{e}^{-\mathrm{i}(m-n+2 k)(\pi-q)}}{\mathrm{i}(m-n+2 k)} & \text { if } m-n+2 k \neq 0 \\
2(\pi-q) & \text { if } m-n+2 k=0\end{cases} \\
& +\mathrm{e}^{\mathrm{i}(m-n) q} \begin{cases}\frac{\mathrm{e}^{\mathrm{i}(m+n+2 k)(\pi-q)}-\mathrm{e}^{-\mathrm{i}(m+n+2 k)(\pi-q)}}{\mathrm{i}(m+n+2 k)} & \text { if } m+n+2 k \neq 0 \\
2(\pi-q) & \text { if } m+n+2 k=0 .\end{cases}
\end{aligned}
$$

\section{Appendix B. Derivation of the Husimi distribution function}

Concentrating on the integral

$$
\int_{0}^{2 a} \mathrm{e}^{-s(x-q)^{2} / 2 \hbar} \mathrm{e}^{-\mathrm{i} p x / \hbar}\left(\mathrm{e}^{\frac{\mathrm{i} n x x}{2 a}}-\mathrm{e}^{-\frac{\mathrm{i} n x x}{2 a}}\right) \mathrm{d} x
$$

which can be rewritten as

$$
\int_{0}^{\pi} \mathrm{e}^{-\frac{s(x-q)^{2}}{2 \hbar}} \mathrm{e}^{-\frac{i p x}{\hbar}} \mathrm{e}^{\frac{i n \pi x}{2 a}} \mathrm{~d} x-\int_{0}^{\pi} \mathrm{e}^{-\frac{s(x-q)^{2}}{2 \hbar}} \mathrm{e}^{-\frac{i p x}{\hbar}} \mathrm{e}^{-\frac{i n \pi x}{2 a}} \mathrm{~d} x
$$

Thus

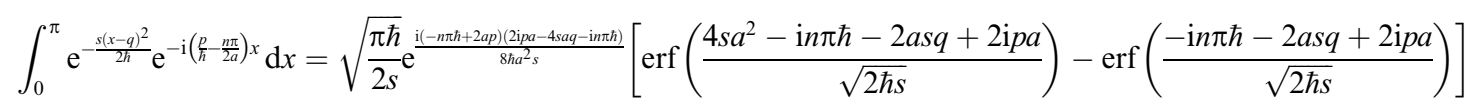

and

$$
\int_{0}^{\pi} \mathrm{e}^{-\frac{s(x-q)^{2}}{2 \hbar}} \mathrm{e}^{-\mathrm{i}\left(\frac{p}{\hbar}+\frac{n \pi}{2 a}\right) x} \mathrm{~d} x=\sqrt{\frac{\pi \hbar}{2 s}} \mathrm{e}^{\mathrm{i}(n \pi \hbar+2 a p)(2 i p a-4 s a q+i n \pi \hbar t)}\left[\operatorname{erf}\left(\frac{4 s a^{2}+\mathrm{i} n \pi \hbar-2 a s q+2 \mathrm{i} p a}{\sqrt{2 \hbar s}}\right)-\operatorname{erf}\left(\frac{\mathrm{i} n \pi \hbar-2 a s q+2 \mathrm{i} p a}{\sqrt{2 \hbar s}}\right)\right] .
$$

\section{References}

[1] Casati G, Chirikov BV, Ford J, Izrailev FM. Lect Notes Phys 1979;93:334.

[2] Chirikov BV. Phys Rep 1979;52:263.

[3] Quantum billiards. J Stat Phys 1995;83 [special issue].

[4] Zaslavsky GM, Zakharov MYu, Sagdeev RZ, Usikov DA, Chernikov AA. Zh Eksp Teor Fiz 1986;91:500.

[5] Zaslavsky GM, Zakharov MYu, Sagdeev RZ, Usikov DA, Chernikov AA. Sov Phys JETP 1986;64:294.

[6] Chernikov AA, Sagdeev RZ, Usikov DA, Zakharov MYu, Zaslavsky GM. Nature 1987;326:559.

[7] Chernikov AA, Sagdeev RZ, Zaslavsky GM. Physica D 1988;33:65.

[8] Zaslavsky GM, Sagdeev RZ, Usikov DA, Chernikov AA. Weak chaos and quasi-regular patterns. Cambridge: Cambridge University Press; 1991. 
[9] Berman GP, Rubaev VY, Zaslavsky GM. Nonlinearity 1991;4:543.

[10] Lichtenberg AJ, Wood BP. Phys Rev A 1989;39:2153.

[11] Dana I, Amit M. Phys Rev E 1995;51:R2731.

[12] Daly HV, Heffernan DM. Acta Phys Pol A 1996;89:571.

[13] Borgonovi F. Phys Rev Lett 1998;80:4653.

[14] Hu B, Li B, Liu J, Gu Y. Phys Rev Lett 1999;82:4224.

[15] Sankaranarayanan R, Lakshminarayan A, Sheorey VB. Phys Lett A 2001;279:313.

[16] Sankaranarayanan R, Lakshminarayan A, Sheorey VB. Phys Rev E 2001;64:046210.

[17] Sankaranarayanan R, Sheorey VB. Phys Lett A 2005;338:288.

[18] Ott E. Chaos in dynamical systems. Cambridge: Cambridge University Press; 1993.

[19] Steck AA, Milner V, Oskay W, Raizen MG. Phys Rev E 2000;62:3461.

[20] Geisel T, Zacherl A, Radons G. Phys Rev Lett 1987;59:2503.

[21] Satija II, Sundaram B. Phys Rev Lett 2000;84:4581.

[22] Richtmyer FK, Kennard EH, Cooper JN. Introduction to modern physics. 6th ed. New Delhi: Tata McGraw-Hill; 1991.

[23] Kilbane D, Cummings A, O' Sullivan G, Heffernan DM. Quantum statistics of a kicked particle in an infinite potential well, Chaos, Solitons \& Fractals, in press, doi:10.1016/j.chaos.2006.01.010.

[24] Dwight HB. Tables of integrals and other mathematical data. New York: The Macmillan Company; 1968.

[25] Izrailev FM. Phys Rep 1990;196:299.

[26] Rechester AB, White RB. Phys Rev Lett 1980;44:1586.

[27] Rechester AB, Rosenbluth MN, White RB. Phys Rev A 1981;23:2664.

[28] Shepelyansky DL. Theor Math Phys 1982;49:925.

[29] Shepelyansky DL. Physica D 1987;28:103.

[30] Wigner EP. Phys Rev 1932;40:749.

[31] Lee H-W. Phys Rep 1995;259:147.

[32] Husimi K. Prog Phys Math Soc Jpn 1940;22:264.

[33] Rajagopal AK. Phys Rev A 1983;27:558.

[34] Kells G, Twamley J, Heffernan DM. Phys Rev E 2004;70:015203(R).

[35] Shin J-Y, Lee H-W. Phys Rev E 1994;50:902. 